drugs had used consist of clopidogrel, aspirin, GP IIb /IIIa receptor antagonist tirofiban and heparin. The first day after operation, it had occurred severe thrombocytopaenia and skin ecchymosis. While took out of all anticoagulant and antiplatelet drugs and transfused platelet, the platelet count returned to normal after one week. After administered again with clopidogrel, aspirin and low molecular weight heparin, the platelet count maintained normal and skin ecchymosis subsided. So this is a case of drug-induced thrombocytopaenia, but we need to identify which kind of drugs led to thrombocytopaenia. In clinical applications, there is a certain incidence of GPIIb /IIIa receptor antagonist-induced thrombocytopaenia (GIT). Some studies suggested that the sensitivity and specificity had not been established to test the related antibodies currently in the clinical practice. So clinical diagnosis of GIT base on the relationship between drug use and time of event. ${ }^{1}$ Since heparin have been used in our case at the same time, it should exclude the possibility of heparin -induced thrombocytopaenia (HIT). ${ }^{2}{ }^{3}$ HIT often occurs about $5-10$ days after the administration of heparin and reaches the diagnosis lever after $7-14$ day. ${ }^{4}$ The incidence of Clopidogrel-induced thrombocytopaenia was $0.2 \%$, which mostly occurs within 2-3 months after taking medicine and is often manifested as thrombotic thrombocytopenic purpura. ${ }^{5}$ It don't support that thrombocytopaenia related to these two antiplatelet drugs through the detection of platelet and observation on drugs and drugs used time in our case. This case is fairly considered the side effects of tirofiban, which is used before the operation. The incidence of tirofiban -induced thrombocytopaenia is $0.1 \%-0.5 \%$. Tirofiban can cause thrombocytopaenia, which, accordingly, can cause bleeding events in foreign reports. China has been reported that tirofiban-induced thrombocytopaenia occurred during the $24 \mathrm{~h}$ after taking medicine while the platelet number decreased to $25 \times 10^{9} / 1 .^{6}$ The mechanisms of GIT is not yet entirely clear and autoimmune response may be the major cause generally. ${ }^{17}$ GP IIb /IIIa receptor antagonist could induce GP receptor conformational change and form new antigentic determinants, which are recognised and bound by plasma antibody and are cleared from the blood lastly. Lessons Learned: Once patients, especially performed PCI due to acute coronary syndrome, are used GP IIb/IIIa antagonists, it should closely monitor the platelet count and observe the skin ecchymosis, haematuria, gastrointestinal bleeding and other performance. Severe thrombocytopaenia can cause fatal brain haemorrhage and massive haemorrhage of gastrointestinal tract. Above all we should review routine blood test to detect GIT early within 2-4 h after using GP IIb /IIIa antagoniste. When it happens, the GP IIb /IIIa antagonists should be immediately suspended and the patients could be treated by transfusing platelets and $\gamma$-globulin, which is often effective.

\section{e0331 EFFECTS OF SHEN SONG YANG XIN CAPSULE FOR TREATMENT OF CARDIAC ARRHYTHMIA: A SYSTEMATIC REVIEW}

doi:10.1136/hrt.2010.208967.331

Hu Hao, Tang Hai-Qin, Li Jie-Hua, Yang Lin-Lin, Tang Wen-Juan, Zhou Zhong-Ran. Department of Geriatrics, The First Affiliated Hospital of Anhui Medical University

Objective To evaluate the efficacy and safety of Shen Song Yang Xin Capsule for treatment of cardiac arrhythmia.

Methods Randomized controlled trials (RCTs) were searched from the following electronic databases: Wanfang, CNKI, CBM, Vip, PubMed, The Cochrane Library. Quality assessment and data extraction were conducted by two reviewers independently. Disagreement was resolved through discussion. All data were analysed by using Review Manager 5.0 software.

Results 13 studies involving 1896 participants involving. Metaanalysis results showed that, compared with control, (1) the efficacy, Shen Song Yang Xin Capsule is better than propafenone
$[R R=0.42,95 \%$ CI (0.28 to 0.62)], mexiletine $[R R=0.34,95 \% \mathrm{CI}$ (0.21 to 0.56)], no significant difference between Shen Song Yang Xin Capsule and amiodarone $[\mathrm{RR}=0.80,95 \%$ CI (0.57 to 1.14)]. Total efficacy for treatment of cardiac arrhythmia is $R R=0.54,95 \%$ CI (0.42 to 0.68). (2) The safety, Shen Song Yang Xin Capsule is no worse than the control in inducing of cardiac arrhythmia $[R R=0.06$, 95\% CI (0.02 to 0.15)], there is no significant difference between Shen Song Yang Xin Capsule and the control in inducing of gastrointestinal adverse reaction $[R R=0.84,95 \%$ CI (0.58 to 1.23$)]$. Conclusion Compared with the current anti-arrhythmic medicine, Shen Song Yang Xin Capsule is no worse than the current antiarrhythmic medicine, lower in the rate of inducing cardiac arrhythmia, and there is no significant difference between Shen Song Yang Xin Capsule and the current anti-arrhythmic medicine in inducing of gastrointestinal adverse reaction. For the restrictions of the quality of the studies, the evaluation of anti-arrhythmic effects look forward to more high-quality RCT to further evaluation.

\section{E0332 TONGXINLUO CAPSULE FOR CORONARY HEART DISEASE: A SYSTEMATIC REVIEW}

doi:10.1136/hrt.2010.208967.332

Zhou Zhong-Ran, Tang Hai-Qin, Li Jie-Hua, Yang Lin-Lin, Hu Hao. Department of Geriatrics, The First Affiliated Hospital of Anhui Medical University, Hefei, China

Objective To assess the effectiveness and safety of tongxinluo capsule for coronary heart disease.

Methods Trials were located electronic searches of the Cochrane Library (Issue 4, 2010), PubMed (1966 to April 2010), Wangfang (1984 to April 2010), CNKI (1979 to April 2010), VIP (1989 to April 2010), and CBM (1978 to April 2010). Randomised controlled trials (RCTs) and quasi-RCTs of tongxinluo capsule for coronary heart disease were included. Quality assessment and data extraction were conducted by two reviewers independently. Disagreement were resolved through discussion. All data were analysed using Review Manager 5.0.

Results 13 studies involving a total of 1496 participants met the inclusion criteria. Meta analysis results showed that: compared with nitrate esters, tongxinluo capsule for the coronary heart disease group had superiority in many aspects such as amelioration according to curative effect: tongxinluo capsule is better than isosorbide dinitrate [RR 0.50 and $95 \%$ CI 0.36 to 0.70 ], than isosorbide mononitrate [RR 0.19 and $95 \%$ CI 0.12 to 0.30 ], total efficacy [RR 0.34 and $95 \%$ CI 0.26 to $0.44, p<0.00001$ ], the two groups had significant difference; Effectiveness according to EKG: tongxinluo capsule is better than isosorbide denigrate [RR 0.55 and $95 \%$ CI 0.46 to 0.66 ], than isosorbide mononitrate [RR 0.58 and $95 \%$ CI 0.48 to 0.70 ], total efficacy [RR 0.56 and $95 \%$ CI 0.49 to 0.64 , $\mathrm{p}<0.00001]$, the two groups had significant difference; the tongxinluo group has a lower rates of adverse effect than itrate esters group. Meta-analysis results showed that the incidence rates of adverse effect [RR 0.33 and $95 \%$ CI 0.20 to 0.53], p<0.00001], the two groups had significant difference.

Conclusion Now we have evidence to indicate that tongxinluo capsule can improve curative effect no worse than isosorbide dinitrate or isosorbide mononitrate, and have a lower rates of adverse effect. But more large scale multi center randomised trials are still needed.

\section{e0333 EFFECTS OF OILIOIANGXIN CAPSULE ON CHRONIC CONGESTIVE HEART FAILURE IN PATIENTS}

doi:10.1136/hrt.2010.208967.333

Mei Ding, Ping Yang. China-Japan Union Hospital of Jilin University, Cardiovascular Department

Objective To study the clinical effect of Qiliqiangxin capsule on chronic congestive heart failure (CHF) in patients for two weeks. 
Methods 64 CHF patients were randomised equally into two groups. All were treated with the basic conventional treatment. Additionally, $1.2 \mathrm{~g}$ of $\mathrm{OC}$ was given three times a day to the therapy group, while no additional treatment was given to the control group. During the treatment, body weight, urine output, sweat secretion and $6 \mathrm{~min}$ walking distance were observed in the two groups. The dosage of cedilanid and furosemide for the first week and second week in the two groups was analysed. The levels of serum $\mathrm{Na}^{+}$in two groups were respectively measured on the second, eighth and fifteenth hospital day. During the treatment, the sideeffect and compliance were observed.

Results 1 and 2 weeks after treatment, the number of sweat secretion was less in two groups, but the decrement was more significant in the therapy group than in the control group $(p<0.05)$. One weeks later, the urine output was more than that before $(p<0.05)$, body weight became less $(p<0.05)$, and 6 min walking distance was better than before, but there are all no significant differences between two groups ( $p>0.05) .2$ weeks later, in both groups, the urine output was more than that before; body weight became less; 6 minwalking distance was better than before. All these differences are significant differences statistically $(p<0.05)$. And there are significant differences about urine output and 6 min walking distance between two groups statistically $(\mathrm{p}<0.05)$; about body weight between two groups there is no significant difference $(p>0.05)$. There are no significant differences about the dosage of lanatoside $\mathrm{C}$ and furosemide in the first and second week between two groups statistically ( $p>0.05)$. The level of serum $\mathrm{Na}^{+}$on the eighth hospital day was less than before in both groups $(p<0.05)$, but there are no significant differences between two groups statistically ( $p>0.05$ ); on the fifteenth day, there is almost no change about that of the therapy group compared with that on the eighth day $(p>0.05)$; on the contrary, there is a significant difference in the control group statistically $(p<0.05)$; and there is a significant difference between two groups statistically $(p<0.05)$. During the period of using the capsule, the 33 patients have not any uncomfortable complain about this drug and nobody stop using this capsule.

Conclusion The effect of $\mathrm{OC}$ about releasing hyperidrosis in a short time is conspicuous. It can play the role in a short time. There is no obvious effect on body weight. The short-term effects of $\mathrm{QC}$ about improving urine output and $6 \mathrm{~min}$ walking distance are not significant, but it plays this role during the second week gradually. QC can't reduce the dosage of cedilanid and furosemide. OC has the effect of keeping the level of serum $\mathrm{Na}^{+}$to some extent. During the observation, the tolerance of this capsule is good, and there is no side effect observed.

\section{e0334 INVESTIGATION FOR EFFECTS OF SOTALOL COMBINED WITH PROPAFENONE TO TREAT PAEDIATRIC ATRIAL TACHYCARDIA}

doi:10.1136/hrt.2010.208967.334

Li Xiaomei, Zhang Yan, Zhang Yi, Bao Min, Lu Ping. First Hospital of Tsinghua University pediatric Cardiac Department

Objects To evaluate the efficacy, safety, selection and combination program of anti-arrhythmic drugs to treat paediatric atrial tachycardia.

Methods 42 children (male 19, female 23, age 7 days-14 years) diagnosed of atrial tachycardia were treated by oral anti-arrhythmic drugs. $24 \mathrm{~h}$ holter monitoring and wireless remote realtime monitoring system were used to evaluate the efficacy. Cardiac function was evaluated by echocardiogram. Side effects of drugs were closely detected during the whole process.

Results 21 cases were firstly treated by single sotalol, none were completely cured, 20 were partially cured (95.2\%), 1 was ineffective
(4.8\%). 41 cases were finally treated by sotalol plus propafenone, 30 were completely cured (73.2\%), 9 were partially cured $(21.9 \%)$ and 2 were ineffective $(4.9 \%)$. The effective dose of sotalol was $5.4-5.7 \mathrm{mg} / \mathrm{kg} . \mathrm{d}$, and the effective dose of propafenone was 9-10.5 mg/kg.d. 5 cases of incessant atrial tachycardia complicated by tachycardiomyopathy $(5 / 17,29.4 \%)$ were treated by amiodarone plus metroprolol, 1 was completely cured and the others' cardiac function were obviously improved after treatment although none of them were completely cured, they all reverted to sotalol plus propafenone while cardiac function became nearly normal. Injury of thyroid function happened in 3 cases after taking oral amiodarone for 3-8 months and all recovered during 1 month after withdrawing the drug. During the 1-14 months of follow up for the 31 cases completely cured, only 1 case of intermittent atrial tachycardia recurred at No.8 month after withdrawing the drug.

Conclusion 1. Sotalol is a safe and effective anti-arrhythmic drug which can be used to treat paediatric atrial tachycardia, effective dose is $4.89-5.71 \mathrm{mg} / \mathrm{kg} . \mathrm{d}$; 2 . Combination use of sotalol plus propafenone is obviously more effective than single use of sotalol with satisfactory safety; 3 . Combination use of sotalol plus propafenone is safer and more effective than combination use of amiodarone plus metroprolol. 4. Amiodarone might lead to thyroid function injury, and can be used as transitional drug for patients diagnosed of tachycardiomyopathy.

\section{e0335 ROLE OF OT INTERVAL DYNAMICITY IN PREDICTING SUDDEN DEATH IN PATIENTS WITH IDIOPATHIC DILATED CARDIOMYOPATHY}

doi:10.1136/hrt.2010.208967.335

Mingwei Bao, Tuantuan Tan, Shengbo Yu, Kui Chen, Congxin Huang. Renmin Hospital of Wuhan University

Objective To investigate the role of OT interval dynamicity in predicting sudden death in patients with idiopathic dilated cardiomyopathy (DCM).

Methods 55 patients with DCM (DCM group) and 27 health people (Control group, Con) were enrolled according to certain criteria. Investigations included history collection, clinical examination, echocardiography, ECG and $24 \mathrm{~h}$ ambulatory ECG. Following indexes were determined, left ventricle end diastolic dimension (LVEDD), left ventricle ejection fraction (LVEF), OT dispersion (QTd), SDNN, the slope of OT/RR plots of the linear regression, ventricular premature beats (VPB) and non-sustained ventricular tachycardia (NSVT). Patients with DCM were followed-up until all cause death.

Results Comparing with Con group, the LVEDD, OTd, VPB/24 h, NSVT/24 h, QTe/RR slope and OTp/RR slope were higher in DCM group, while LVEF and SDNN were lower in DCM group $(p<0.05)$. The LVEDD, LVEF, OTd, SDNN, OTe/RR slope and OTp/RR slope were significant different among DCM sudden death group, DCM non sudden death group and Con group $(\mathrm{p}<0.05)$. Comparing between DCM sudden death and non sudden death group, LVEF, SDNN, QTp/RR slope and OTp/RR slope shown significant difference $(p<0.05)$. Comparing between DCM with NSVT and without NSVT group, LVEF, OTd, VPB/24 h, OTp/RR slope and QTp/RR slope shown significant difference $(p<0.05)$. The sudden death rate of DCM patients with $\mathrm{QTe} / \mathrm{RR}$ slope $\geq 0.210$ was higher than those $<0.210$ ( $54.5 \%$ vs $21.1 \%, \mathrm{p}<0.05)$. Sudden death rate of QTp/RR slope $\geq 0.190$ was higher than those $<0.190(52.2 \%$ vs $21.9 \%, \mathrm{p}<0.05)$. The sudden death rate of DCM patients with both LVEF $35 \%$ and NSVT+ was $62.5 \%$. Combining $\mathrm{OTe} / \mathrm{RR} \geq 0.210$ with NSVT+ or LVEF $35 \%$, the sudden death rates were $62.5 \%$ or $66.7 \%$. Combining QTp/RR $\geq 0.190$ with NSVT + or LVEF $35 \%$, the sudden death rates were $66.7 \%$ or $61.5 \%$. Combining QTe/ $\mathrm{RR} \geq 0.210$ or $\mathrm{QTp} / \mathrm{RR} \geq 0.190$ with NVST+ and LVEF $35 \%$, the sudden death rates were $77.8 \%$ or $70.0 \%$. 